\title{
Intermédialités
}

Histoire et théorie des arts, des lettres et des techniques

Intermediality

History and Theory of the Arts, Literature and Technologies

\section{Les jeux narratifs des remakes de Pierre Huyghe}

\section{Marie Fraser}

Numéro 9, printemps 2007

Jouer

Playing

URI : https://id.erudit.org/iderudit/1005529ar

DOI : https://doi.org/10.7202/1005529ar

Aller au sommaire du numéro

\section{Éditeur(s)}

Centre de recherche sur l'intermédialité

ISSN

1705-8546 (imprimé)

1920-3136 (numérique)

Découvrir la revue

Citer cet article

Fraser, M. (2007). Les jeux narratifs des remakes de Pierre Huyghe.

Intermédialités / Intermediality, (9), 45-58. https://doi.org/10.7202/1005529ar

\section{Résumé de l'article}

Les liens entre jeu et narrativité ont fait l'objet de plusieurs analyses au cours des dernières décennies, surtout lorsqu'il s'agit de resituer le rôle et l'engagement du lecteur (du spectateur) en fonction de la libre activité du jeu. L'idée d'un registre ludique développée par Jacques Rancière dans Malaise dans l'esthétique (2004) diffère considérablement des conceptions structuraliste et herméneutique. Ouvrant plutôt le débat sur l'esthétique et la position critique de l'art, Jacques Rancière définit le jeu comme un " processus de délégitimation ». Les remakes de films que Pierre Huyghe a réalisés au cours des années 1990 représentent un beau cas d'intermédialité où la notion de jeu intervient pour déjouer des structures narratives déjà existantes, pour les détourner et les prolonger au-delà d'elles-mêmes. Le remake offre par la reduplication une critique des systèmes narratifs dont il se veut pourtant la reprise, de même que de l'industrie cinématographique. Cet article analyse ces jeux narratifs dans la perspective d'un renouvellement du récit et de la narrativité qui a cours aujourd'hui dans l'art contemporain.
Ce document est protégé par la loi sur le droit d'auteur. L'utilisation des services d’Érudit (y compris la reproduction) est assujettie à sa politique d'utilisation que vous pouvez consulter en ligne.

https://apropos.erudit.org/fr/usagers/politique-dutilisation/ 


\title{
Les jeux narratifs des remakes de Pierre Huyghe
}

\author{
Marie Fraser
}

L

es liens entre jeu et narrativité ont fait l'objet de plusieurs analyses au cours gagement du lecteur (du spectateur) en fonction de la libre activité du jeu. Les remakes de films que Pierre Huyghe a réalisés au cours des années 1990 engagent des jeux sur la narrativité qui permettent de penser ces deux notions non pas tant dans un rapport d'opposition mais comme une stratégie pour ouvrir et resituer le récit, autrement dit pour appliquer à la narrativité le potentiel d'ouverture que l'on attribue habituellement au ludique. Invitant à reconsidérer les mécanismes narratifs des systèmes prédéterminés dont il se veut la reprise, le remake deviendrait même prétexte à jouer sur la narrativité. Roland Barthes fut peut-être le premier à attribuer cette stratégie critique au concept de jeu lorsqu'il souligne, dans «La mort de l'auteur», qu' « un code ne peut se détruire, on peut seulement le “jouer" ». Le jeu pourrait ainsi être compris comme un procédé permettant de déjouer des structures narratives déjà existantes et de les remettre en question. Par leurs jeux sur la structure narrative, les remakes de Pierre Huyghe qui seront étudiés dans cet article incitent à revoir l'étude de la narration selon les paramètres de la narratologie. Comme si le jeu pouvait provoquer un effet de renversement, de basculement, obligeant à repenser l'analyse de la narrativité: au lieu de partir du récit pour aller vers sa structure, il s'agirait d'élucider cette structure pour l'ouvrir et non plus pour la formaliser. L'intermédialité est au cœur de cette problématique du jeu et de la narrativité. Le remake est certes une forme de remédiation, mais dont les effets critique et d'ouverture se trouvent ici enclenchés par la mise en exposition du cinéma, ce que Jean-Christophe Royoux

1. Roland Barthes, «La mort de l'auteur» [1968], dans Le bruissement de la langue. Essais critiques IV, Paris, Seuil, 1984, p. 65. 
a nommé avec justesse le «cinéma d'exposition²». Le passage du cinéma à l'exposition permet de spatialiser l'expérience narrative et temporelle de l'image jusqu'ici confinée à l'intérieur des normes et du dispositif cinématographiques. Ce changement de dispositif, de média, joue un rôle crucial dans la reformulation de la narrativité que l'on retrouve chez Pierre Huyghe. C'est même en tant que processus d’intermédialité que le remake se révélera capable de jouer sur et avec la structure narrative et temporelle du récit cinématographique. Le fait de refilmer une scène ou de rejouer un film intégralement, comme nous souhaitons le montrer ici, permet en fait de poursuivre et d'étendre la narrativité au-delà des codes, des moyens et des procédés techniques cinématographiques.

Depuis 1995, Pierre Huyghe a réalisé une série d'installations qui s'approprient toutes sortes de films: Atlantic (Ewald André Dupont, 1929), Poltergeist (Steven Spielberg, 1982), Snow White and the Seven Dwarfs (Walt Disney, 1937), Dog Day Afternoon (Sydney Lumet, 1975), des thrillers comme Rear Window (Alfred Hitchcock, 1954) ainsi que des films du cinéma d'auteurs, comme Uccellacci e uccellini (Des oiseaux petits et grands, Pier Paolo Pasolini, 1966) et Der Amerikanische Freunde (L'ami américain, Wim Wenders, 1977). Les remakes produits à partir de ces films se penchent sur différents aspects du dispositif cinématographique (la durée, le montage, les ellipses, les jeux de rôle et d'acteur) pour interroger le fonctionnement de la narrativité au cinéma. Malgré l'évidence d'une telle position critique à l'égard de la narrativité, cette approche n'est pas pour autant déconstructive. Le remake permet au contraire, comme il sera ici démontré, d'ouvrir une zone d'expansion et de prolifération narratives sans précédent pour les théories du récit. Il inscrit un film (un récit) dans un processus qui produit d'autres films, d'autres récits, jusqu'à prolonger la fiction dans le réel. L'enjeu reste donc encore de produire du récit mais à partir d'une réinscription de la fiction dans la réalité. Cette zone d'expansion et de prolifération narrative laisserait libre cours aux conflits entre la fiction et la réalité, jusqu'à introduire une critique de l'industrie cinématographique. Cet espace conflictuel du jeu rejoint l’idée d’un registre ludique, développé par Jacques Rancière dans Malaise dans

2. Jean-Charles Royoux, «Remaking Cinema. Les nouvelles stratégies du remake et l'invention du "cinéma d'exposition” ", dans Véronique Goudinoux, Michel Weemans, Luis Perez-Oramas (dirs.), Reproductibilité et irreproductibilité de l'oeuvre d'art, Bruxelles, Éditions La Lettre volée, 2001, p. 215-216. Notons, par contre, que Jean-Christophe Royoux n'aborde pas le «cinéma d'exposition» en fonction de la notion d'intermédialité, mais comme manière de nommer l'intérêt que représente le cinéma chez plusieurs artistes depuis les années 1990. 
l'esthétique3. Cherchant à ouvrir le débat entre l'esthétique et la position critique de l'art, Jacques Rancière envisage le concept de jeu comme un «processus de délégitimation ».

Une des principales stratégies que Pierre Huyghe a explorée pour créer cet effet d'expansion et de prolifération à partir de structures narratives déjà existantes (et fermées sur elles-mêmes), c'est de prolonger le temps narratif dans le temps réel et vécu du monde, selon l'expression de Paul Ricœur. Rappelons qu'un des principes fondateurs de la narratologie est de séparer les deux pour maintenir l'unité narrative et temporelle du récit à l'intérieur d'une structure logique. Une attention particulière sera consacrée ici à The Third Memory (1999), car cette œuvre permet de traiter cette problématique dans toute sa complexité. Le remake a pour point de départ un film déjà basé sur un fait réel, Dog Day Afternoon de Sydney Lumet, et fait appel au véritable «auteur» du crime pour raconter sa propre histoire.

La mise en scène d'une réalité donnée est d'ailleurs le premier procédé de reprise et de doublage que Pierre Huyghe a utilisé avant d'expérimenter des modèles cinématographiques. Ses Billboards (1994) réalisés sur des chantiers de construction parisiens sont souvent considérés comme ses premiers remakes. Barbès-Rochechouart, Billboard, Paris, 1994, par exemple, confronte la réalité d'un site en construction avec les ouvriers au travail à sa mise en scène photographique par des acteurs. Présenté sur les lieux mêmes du chantier, le panneau publicitaire double le réel mais joue sur les écarts de temps et de perception entre la prise de vue photographique et le site réel. Assez curieusement pour la photographie, la mise en scène produit une expérience temporelle qui fonctionne à l'inverse du «ça-a-été» que Roland Barthes associe à la photographie ${ }^{4}$. Au lieu de référer à un moment passé fondateur, elle ouvre au contraire un espace potentiel.

Alors qu'un artiste comme Douglas Gordon manipule des «vrais » films (en l'occurrence Psycho [1960] d'Alfred Hitchcock5), Pierre Huyghe les recrée de toutes pièces avec ses propres acteurs, décors, caméra, etc. Déplacement

3. Voir Jacques Rancière, "Problèmes et transformation de l'art critique », dans Malaise dans l'esthétique, Paris, Éditions Galilée, coll. «La philosophie en effet», 2004, p. $65-84$.

4. Roland Barthes, La chambre claire. Note sur la photographie, Paris, Cahiers du Cinéma, Éditions Gallimard, Seuil, 1980.

5. Je pense ici à 24 Hours Psycho de Douglas Gordon, réalisé en 1986. L'œuvre est une projection du film Psycho d'Alfred Hitchcock sur un écran translucide suspendu au milieu d'un espace vide. L'artiste a supprimé le son et ralenti le film de sorte que sa durée originale a été rallongée à vingt-quatre heures. Douglas Gordon procède à une expansion temporelle qui dissout les mécanismes narratifs du suspense. 
temporel, changement d'époque et réactualisation demeurent encore des moteurs du remake. Mais reprendre un film suppose aussi, et plus fondamentalement, de réactiver, de prolonger et de déplacer un modèle narratif et temporel pour l'inscrire dans un autre contexte. Dans les œuvres de Pierre Huyghe dont il est ici question, cette réactualisation déclenche une reformulation de la narrativité qui repose d'abord sur la structure. Le remake impliquerait en fait de remettre en jeu les structures narratives et temporelles des films qu'il s'approprie. La structure est le point de départ, comme l'explique Pierre Huyghe:

If I use a structure that I re-present, I do so in order to confront them in a context which is different each time; then they allow me to describe a different event. It's an articulation of different structures, a reading through displacement. Instead of adding new structures, it may be preferable to consider the existing ones and to ask which stories they can produce. The narrative moves through this succession of transfers. How to inhabit these structures? What are their potentials? What movements can I give them? It is this movement, this vector, which should be watched ${ }^{6}$.

Pour son premier film, Les incivils (1995), qui est une reconstitution de scènes de Uccellacci e uccellini de Pier Paolo Pasolini, Pierre Huyghe est retourné sur les lieux de tournage et les sites de location avec l'acteur fétiche du réalisateur, Ninetto Davoli, à qui il avait proposé de réincarner son propre rôle trente ans plus tard. Entre passé et présent, entre fiction et réalité, les scènes refilmées sont intercalées entre des scènes de la version originale et des moments du tournage. Les incivils se construit ainsi dans des espaces transitoires et aléatoires à l'intérieur d'un cadre narratif donné. Dans ce film, comme dans tous les autres où les acteurs sont appelés à rejouer une scène du film original, ce qui implique nécessairement une nouvelle temporalité, le remake servirait à «redonner pour un temps une réalité aux acteurs, au tournage, à tout le processus du film d'origine». Il est «un objet neuf qui part du film et qui revient au réel par le biais d'un dispositif7 $»$.

L'idée de provoquer ce basculement de la fiction pour la confronter à la réalité est aussi présente dans Remake (1994-1995), qui est la reprise intégrale, plan par plan, de Rear Window d'Alfred Hitchcock, un des films cultes de l'histoire du cinéma hollywoodien et un des modèles cinématographiques du suspense (figs. 1-2). Les principaux personnages sont doublés par des interprètes amateurs qui redisent le texte mais sans le mettre en scène. Le film est enregistré dans l'appartement de l'«acteur» qui double le personnage principal, joué par James

6. «Pierre Huyghe interviewed by Jérôme Sans», dans Pierre Huyghe, Vienne, Secession, 1999, [non paginé].

7. Vincent Dieutre, «Pièces jointes pour un procès du temps libre. Dialogue entre Pierre Huyghe et Vincent Dieutre », Lettre du cinéma, n 15, automne 2000, p. 28. 
Stewart (L. B. Jefferies), un photographe de presse immobilisé à la suite d'un accident. Tout se joue dans le moment présent de l'enregistrement: les artifices du cinéma qui permettent la construction du suspense ont laissé place à la spontanéité et à l'amateurisme des interprètes qui répètent mécaniquement le texte sans véritablement le mettre en action, autrement dit sans l'investir de sa dimension psychologique. Les «doubleurs » ont même parfois de la difficulté à se souvenir de leur texte, et la distance qu'ils adoptent face au récit annule la complexité psychologique du film. Le doublage devient ainsi prétexte à expérimenter «un modèle narratif à travers un transfert de contexte. Paris, ici, maintenant,

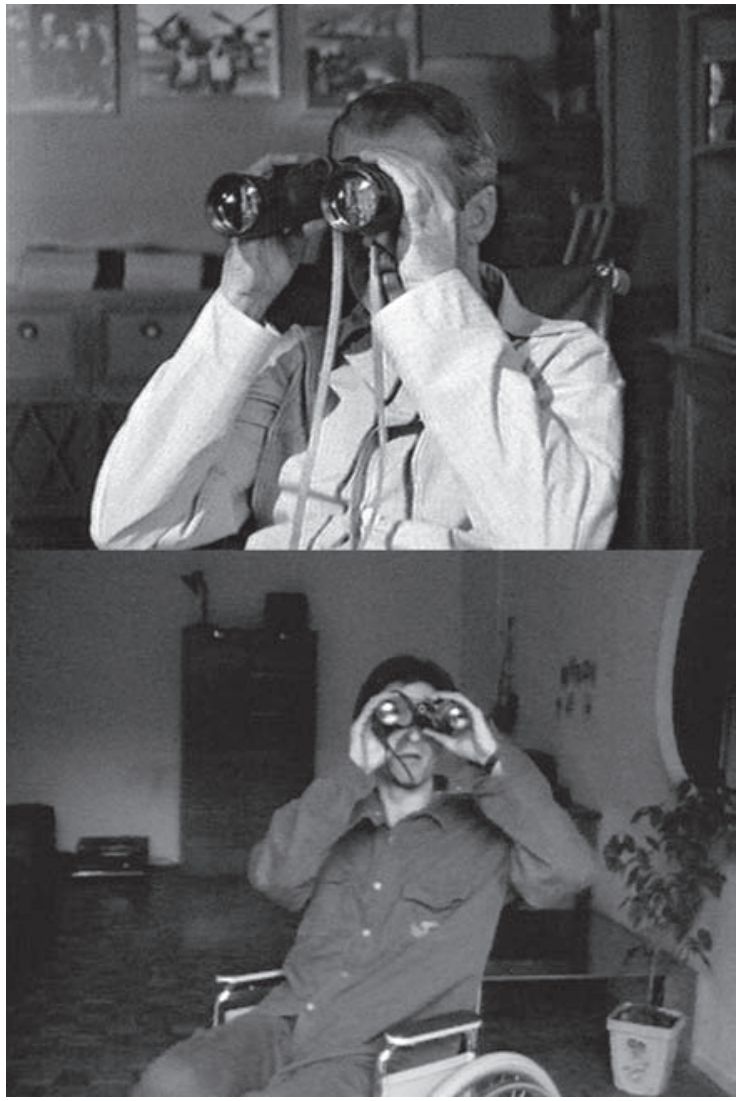

Figs. 1-2. Pierre Huyghe, Remake, 1994-1995, projection vidéo, Hi8/ Master Beta digital, 100 minutes. Avec l'aimable permission de la Galerie Marian Goodman, New York/Paris. 
sans moyen, sans Hollywood ${ }^{8}$ », et «il ne subsiste du film que les structures fon-

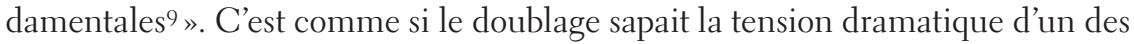
paradigmes les plus notoires du suspense, Rear Window, et qu'il dirigeait l'attention vers la structure filmique, vers les mécanismes narratifs et temporels qui construisent le suspense.

Cette dédramatisation coïncide avec une déconstruction de la linéarité, qui est le moteur du défilement cinématographique, comme l’a remarqué JeanChristophe Royoux à propos de Remake:

[le film] a pour effet de dissocier chaque plan du film du continuum cinématographique. Si une telle interprétation se justifie cependant, ce n'est pas parce qu'il [le remake] arrêterait l'image ou la ralentirait, mais parce que la distance qu'instaure le jeu (ou plutôt l'absence de jeu des interprètes) par rapport à l'intrigue est telle qu'il devient impossible de le percevoir autrement que comme une succession de moments, comme s'il s'agissait d'une suite de morceaux, de tableaux parlants et animés, qui en même temps qu'ils en constituent la trame, décomposent le mouvement du défilement cinématographique. Le «désinvestissement» psychologique du rôle, la dévitalisation du film original, bien que l'intrigue, le découpage et les dialogues semblent avoir été respectés et même strictement reproduits, interdisent ou en tout cas découragent toute attention portée à l'histoire racontée ${ }^{10}$.

Jean-Christophe Royoux n'est pas sensible à l'indétermination entre fiction et réalité qui est créée par la dévitalisation dont il parle. Le remake de Pierre Huyghe confère au récit cinématographique une réalité déjà présente dans le projet original d'Hitchcock, c'est-à-dire de créer une intrigue dont le suspense repose en partie sur son potentiel de réalité, sur la dimension plausible de l'événement que le personnage principal incarne à lui seul. Pierre Huyghe inverse donc ce qui était déjà dans le film Rear Window: si ce dernier joue sur les effets dramatiques que peut engendrer l'éventualité d'une telle situation, le remake dédramatise l'intrigue. Pierre Huyghe pousse donc ainsi la structure du film à son point limite, jusqu'à ce qu'elle bascule complètement dans la réalité et qu'il ne reste de la fiction qu'une allusion au film original.

Dubbing (1996) procède à une dévitalisation similaire, en reconstituant le procédé de doublage du film d'horreur Poltergeist. Cette fois, c'est la trame sonore qui fait l'objet d'une reprise et qui devient l'unique référent visuel. En un seul plan-séquence d'une heure et demie sans montage, Pierre Huyghe a filmé

8. Armelle Leturcq, dans «Remake. My Own Private Psycho. Rencontre autour de Psycho", Cahiers du cinéma, n 532, janvier 1999, p. $4^{8}$.

9. Clarisse Hahn, «Pierre Huyghe», Art Press, n²03, juin 1995, p. 79.

10. Jean-Charles Royoux, «Remaking Cinema. Les nouvelles stratégies du remake et l'invention du "cinéma d'exposition" », p. 221. 
les doubleurs qui répètent le texte en continu, assis les uns à côté des autres dans un studio. Vêtus de jeans et t-shirts, ils n'ont visuellement rien à voir avec les rôles que leurs voix incarnent. Généralement, la production d'un doublage au cinéma s'effectue séparément pour chacune des voix et c'est le travail de montage qui produit la trame sonore avant de la synchroniser avec l'image. En isolant la trame sonore de l'image, le son reste le seul élément constitutif de la narrativité du film. L'image n’a plus aucune fonction diégétique; ce qui est montré, c'est la réalité du processus de doublage qui, elle, à son tour, expose le fonctionnement de l'intrigue.

Versions Multiples (Atlantic-Atlantik-Atlantis, 1929), réalisé en 1997, a par la suite exploré les écarts qui surviennent lorsque le doublage nécessite, comme dans les années 1920 et 1930, de rejouer le scénario intégral dans une autre langue en faisant appel à des acteurs étrangers. Les trois versions du film Atlantic sont projetées simultanément côte à côte, en anglais, en français et en allemand. Cette triple projection confronte différentes interprétations d'un même modèle de scénario, les trois dernières heures du Titanic, en rendant évidentes les variations de la réalisation et de l'interprétation, les décalages narratifs et temporels (les langues ne vont pas toutes à la même vitesse: une scène peut prendre plus de temps en français qu'en anglais par exemple, ce qui est généralement le cas), la variabilité des montages et les dissimilitudes entre les jeux d'acteurs et les décors. Quoique les interprétations diffèrent en fonction des particularités des langues, la structure narrative demeure identique, comme opaque aux différences linguistiques et culturelles.

Blanche-Neige Lucie (1997), Ellipse (1998) et The Third Memory radicalisent ce basculement de la fiction dans la réalité en ayant recours aux acteurs des films originaux pour commenter, voire pour rejouer des scènes originales. Les acteurs sortent ainsi de la structure filmique pour en prolonger la narrativité en dehors de l'univers fictionnel cinématographique. Dans Blanche-Neige Lucie, ce retour du personnage fictif à la réalité remet en question le rêve hollywoodien et l'imaginaire fantastique du cinéma. Si plusieurs remakes de Pierre Huyghe engagent le récit cinématographique dans un processus de dédramatisation, ici, il serait plus juste de dire que l'histoire de Lucie Dolène, la voix française du célèbre film d'animation de Walt Disney, Blanche-Neige, conduit vers un désenchantement. Dans un plan-séquence de quatre minutes, Lucie Dolène chantonne l'air de la chanson thème «Un jour mon prince viendra... », alors qu'en sous-titres, une autre histoire apparaît et résonne comme une réminiscence nostalgique du célèbre personnage. Lucie Dolène raconte qu'en prêtant sa voix pour le doublage français du film, elle s'est identifiée au personnage, entrant ainsi pleinement dans l'univers fantastique du récit. Elle relate également la lutte qu'elle a livrée pendant plusieurs années contre Disney Voice Character pour faire reconnaître ses droits. Contrastant avec le son de sa voix fredonnant, les sous-titres ouvrent 
un espace critique où l'univers merveilleux du film se trouve confronté à son contexte réel de production et à sa réalité économique.

Dans Ellipse, Bruno Ganz rejoue en temps réel une des scènes de Der Amerikanische Freunde que Win Wenders avait filmée mais qu'il n’avait pas conservée dans la version finale de son film en 1977. Lacteur principal revient sur les lieux du tournage pour refilmer son propre rôle vingt ans plus tard, autrement dit dans une réalité et une temporalité différentes. Ellipse sort ainsi le récit de sa propre fiction mais, comme l'explique Pierre Huyghe, son remake ajoute une part d'interprétation qui a aussi pour effet de brouiller la réalité :

Ellipse is just one hypothesis of the intermediary moment. It's just one of the many possible versions. The fact that you shift from the young actor to the man today, that he leaves his role to re-enter the original pattern of the film and again becomes the actor he was, produces another ellipse, a temporal one. It is an extra meaning that obscures the reality: even if the signs of the event are weak, they exist ${ }^{11}$.

Le choix de travailler une ellipse permet d'infiltrer la structure narrative et temporelle du film. Au cinéma, l'ellipse a des «fonctions rythmiques, dramatiques et narratives ${ }^{12}$ » permettant de passer rapidement d'un point à un autre de la fiction pour comprimer le temps réel dans le temps narratif. Ellipse inverse ce phénomène essentiel au rythme du film en investissant un des espaces-temps compris entre deux plans-séquences, et en y réinscrivant la durée du temps réel. Cette reconstitution d'une des ellipses du film original n'a pas pour effet de combler cet espace-temps, ce qui renforcerait la clôture du récit, mais d'ouvrir une brèche à l'intérieur de la structure narrative et temporelle. Quelque vingt ans plus tard, l'acteur du film original rejoue donc la scène initialement coupée au montage par Wim Wenders. Partant d'un point à un autre du récit mais également d'un point géographique à un autre, Bruno Ganz traverse le pont de Grenelle entre deux quartiers de Paris; un parcours d'une durée de huit minutes. L'installation utilise un dispositif à trois écrans fonctionnant simultanément, où l'on voit la scène se dérouler en temps réel entre les deux plans-séquences du film (fig. 3). L'idée du remake trouve ici sa place au creux d'un «moment intermédiaire », dans un espace-temps interstitiel inoccupé entre deux scènes, entre deux moments du récit dont la durée n’est pas montrée. La scène refilmée vient réinterpréter cette zone libre non pas pour la réinscrire à l'intérieur du dispositif séquentiel, mais pour décentrer le récit, le faire dévier et le projeter au-delà de

11. Pierre Huyghe, dans SMMoAcôte ouest: Pierre Huyghe - cinema installation et Marie-Ange Guilleminot - watch project and transformation parlor, 23 septembre/2 novembre 1999 [non paginé].

12. François Vanoye, Scénarios modèles, modèles de scénarios, Paris, Nathan, coll. «Nathan-université», 1991, p. 110. 
la trame narrative filmique et le déplacer dans la réalité. Dans L'ami américain, ces zones libres font partie intégrante du dispositif séquentiel ${ }^{13}$. Le film original ne montre pas l'espace-temps compris d'un point à l'autre du parcours, il se reconstruit virtuellement au moment de la scène suivante. L'ellipse est donc déjà au cinéma une zone interstitielle, virtuelle, qui entraîne la narrativité vers autre chose: «Ainsi, Pierre Huyghe prolonge-t-il le travail de réflexion sur le temps que le film de Wenders tenait au centre de son dispositif. En réalisant ce plan-séquence qui identifie le concept à l'image, l'artiste recharge le film d'une nouvelle nappe de temporalité ${ }^{14}$ ». En fait, ce moment du film est réinscrit deux fois dans la durée du temps réel.

Remake, Versions multiples (Atlantic - Atlantik - Atlantis, 1929) et Ellipse

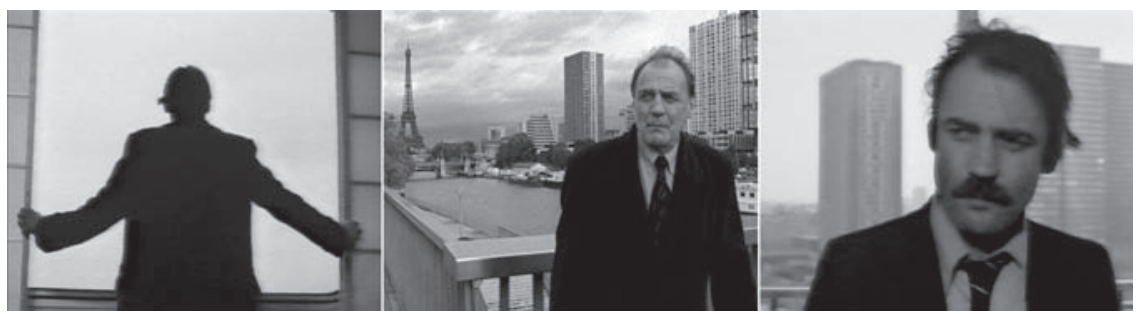

Fig. 3. Pierre Huyghe, Ellipse, 1998, triple projection, S16, Beta digital, son, 13 minutes. Avec l'aimable permission de la Galerie Marian Goodman, New York/Paris.

sont toutes des œuvres fondées sur la présence et le rôle d'acteurs qui commentent ou réinterprètent le film original, en provoquant un prolongement narratif et temporel qui a tendance à pousser la narrativité en dehors du cadre cinématographique. Pierre Huyghe souligne que, dans chacun des cas, il y a une «re-représentation, deux [et même plusieurs] interprétations différentes qui ne produisent pas les mêmes histoires ${ }^{15}$ ». Comme il l'explique en des termes où l'on pourrait aisément reconnaître la pensée de Gilles Deleuze,

[...] it's more interesting to set down very distanced points so that the lines continue to be drawn, and so they are always potential, always offering possible interpretation. [...] There is a double experience, a co-habitation, a back-and-forth movement, a shift between one form to another in the process of becoming ${ }^{16}$.

13. François Vanoye, Scénarios modèles, modèles de scénarios, p. 110.

14. Françoise Parfait, Vidéo: un art contemporain, Paris, Éditions du Regard, 2001, p. 292.

15. Pierre Huyghe dans «Remake. My Own Private Psycho. Rencontre autour de Psycho», p. $4^{8 .}$

16. «Pierre Huyghe interviewed by Jérôme Sans » [non paginé]. 
Dans The Third Memory, l'idée d'ouvrir et de prolonger une structure narrative en la confrontant au réel est encore la plus explicite. Pierre Huyghe a proposé à John Wojtowicz de rejouer sa propre histoire plusieurs années après que Sydney Lumet l'ait portée sur grand écran dans Dog Day Afternoon (fig. 4). Mettant en vedette Al Pacino dans le rôle principal de Sonny Wortzik, Dog Day Afternoon est une adaptation d'un fait divers, le hold-up commis par John Wojtowicz dans une banque de Brooklyn le 22 août 1972. The Third Memory réactive ce récit cinématographique en plus de le réactualiser quelque vingt plus tard. Devant les caméras, John Wojtowicz, le véritable auteur du hold-up, raconte sa propre histoire à partir de deux des scènes stratégiques du film original : la prise d'otages à l'intérieur de la banque ainsi que ses pourparlers avec la police de New York et le FBI. En décrivant l'action dont il était le principal protagoniste, John Wojtowicz ne peut s'empêcher de commenter le film de Sidney Lumet et la façon dont Al Pacino interprète son rôle. Le fait vécu «original» est donc raconté deux fois, mais à deux moments différents, en plus d'être pris dans l'enchevêtrement de la réalité et de la fiction: d'un côté, Al Pacino est dans l'espace et le temps narratifs du film alors que, de l'autre, John Wojtowicz est dans la réalité sans toutefois qu'il puisse complètement se détacher de la fiction. En ajoutant de nouvelles strates narratives mais en périphérie du film, son récit abolit la rupture entre les registres de la fiction et de la réalité. Cette confusion agit à un point tel, comme le soulève Jan Estep en entrevue avec l'artiste, qu'on perd de vue la distinction entre le vrai et le faux, entre le réel et le fictionnel: "there are so many levels to the performance and the film that you're not quite sure what is true or false, subjectively or objectively told ${ }^{17}$ ». En jouant son propre rôle, John Wojtowicz provoque une confusion entre son histoire vécue et son histoire racontée où s'abolissent les frontières de la réalité et de la fiction; celles-ci deviennent de plus en plus difficiles à cerner, voire à définir. Cette indétermination n'empêche pas la narrativité de fonctionner comme on pourrait le penser, au contraire, elle l'ouvre pour que d'autres récits émergent librement.

Le choix du film correspond bien à cette reformulation de la narrativité puisque Dog Day Afternoon reprend un fait vécu pour le configurer avec les moyens filmiques. L'adaptation cinématographique transfère le monde réel dans un monde fictionnel qui a ses propres mécanismes et codes narratifs. Cette médiation exige que l'espace et le temps réels répondent à des règles et à des normes spécifiques pour êtres transposés dans un espace et un temps narratifs logiquement construits. En plus d'être recadré par le support cinématographique, le fait vécu est également contraint par l'industrie, par les normes hollywoodiennes,

17. Jan Estep, «Action! An Interview with Pierre Huyghe», New Art Examiner, vol. $27, n^{\circ} 10$, juillet-août 2000, p. 34 . 


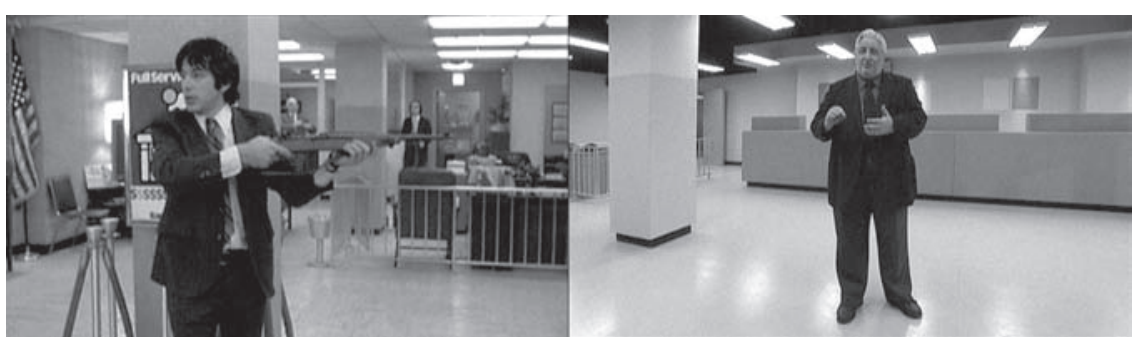

Fig. 4. Pierre Huyghe, The Third Memory, 1999, double projection, Beta digital, vidéo sur moniteur, 9 minutes. Avec l'aimable permission de la Galerie Marian Goodman, New York/Paris.

leurs conditions de scénarisation, de production et de diffusion ${ }^{18}$ et, pourrait-on ajouter, par le genre lui-même - que l'on connaît aujourd'hui sous le vocable de «cinéma vécu » - qui se développe à Hollywood durant les années 1970. C'est d'ailleurs cette industrie que l'auteur du «vrai » crime affronte lorsque, après la sortie du film, il engage des démarches pour «corriger» la fiction et faire valoir son histoire telle qu'elle s'est véritablement déroulée le jour du hold-up.

La réponse à ces questions ne se trouve pas uniquement dans la séparation de la réalité et de la fiction, comme le poserait la narratologie qui insiste pour séparer les deux mondes et les traiter comme des entités séparées, autonomes ${ }^{19}$. L'étude du récit devrait être en mesure d'intégrer cet espace conflictuel entre le réel et la fiction. Il ne s'agit pas seulement de fictionnaliser le réel ou de rendre la fiction plus réaliste. Le mouvement d'aller et de retour entre la fiction et la réalité crée une zone d'expansion et de prolifération narratives qu'il n'est plus possible de ramener à une seule structure narrative. Les jeux sur la narrativité auxquels se livrent les remakes de Pierre Huyghe et, particulièrement ici, avec The Third Memory, permettraient d'intégrer cette zone entre la fiction et la réalité que la narratologie trouve conflictuelle. La notion de jeu rend en effet possible

18. Pour une analyse plus approfondie des conditions de production qui déterminent l'adaptation d'un fait vécu en le transformant en mise en scène dramatique pour répondre au modèle narratif imposé par l'industrie cinématographique hollywoodienne, voir le texte de Jean-Charles Masséra, «La leçon de Stains (Pour une esthétique de la reconstitution)», dans Christine Van Assche et Jean-Charles Masséra (dirs.), Pierre Huyghe: The Third Memory, Paris, Chicago, Centre Georges Pompidou, The Renaissance Society at the University of Chicago, 2000, p. 17-61.

19. Voir notamment l'ouvrage récent de Dorrit Cohn, Le propre de la fiction, trad. Claude Hary-Schaeffer, Paris, Éditions du Seuil, 2001 [1999]. Mais soulignons que pour Paul Ricœur, le problème du récit fictionnel et du récit non fictionnel (l'histoire et le vécu) reste entier malgré l'analyse magistrale et très fouillée qu'il propose dans Temps et récit. 
l'intégration de cet espace-temps conflictuel et permet d'introduire l'idée que dans l'intervalle et le passage entre les deux mondes, il se produit encore de la narrativité. The Third Memory est un bel exemple de récit qui fonctionne d'une manière telle qu'il éveille d'autres aspects relevant à la fois de la fiction et du réel. Ce n'est pas la réalité qui ressort, ce sont ses ambiguïtés. Le jeu permet, mieux que la narrativité, de rendre compte de cette complexité, parce qu'il construit une interface qui agit ici entre plusieurs versions d'un même événement.

Si le film de Sidney Lumet s'élabore d'après un fait vécu, celui de Pierre Huyghe s'élabore d'après sa propre fiction. Les deux récits se croisent et se rencontrent, ils se superposent sans toutefois se dérouler dans le même sens, dans le même espace et dans le même temps, comme une troisième mémoire. The Third Memory tente ainsi de ré-ouvrir l'espace et le temps que Dog Day Afternoon avait refermés. Pierre Huyghe dit qu'il a cherché à provoquer une extension de la fiction à la réalité pour travailler les ouvertures narratives que cette extension peut potentiellement laisser échapper ${ }^{20}$. The Third Memory commence donc là où la fiction s'arrête dans Dog Day Afternoon, là où le réel prend son relais pour prolonger la narrativité au-delà de la structure du récit. Le remake marque ainsi le point de départ de quelque chose d'autre, le point de départ d'un processus qui transforme, en l'interrogeant, le temps et l'horizon narratif du récit ${ }^{21}$.

L'enjeu est double: dans un premier temps, le remake dévoile la structure narrative du film, c'est-à-dire qu'il montre la manière dont un film raconte un événement; dans un second temps, il prolonge le récit au-delà et en deçà de sa structure pour faire émerger d'autres récits, voire d'autres formes de narration. Cette reconsidération de la narrativité repose en majeure partie sur le temps. Dans Dog Day Afternoon, les mécanismes du suspense organisent la temporalité de telle sorte que le film se concentre sur les moments forts et stratégiques de l'événement: d'une prise d'otages d'une dizaine d'heures où l'attente est constante, on passe à une séquence d'une heure et demie où c'est la tension du suspense qui est constante. Comme le suggère Jean-Charles Masséra, la temporalité dans The Third Memory se déroule en dehors « du suspense et de l'organisation des éléments constitutifs du récit»; il s'agit d'un «temps non scénarisable», «indépendant du [...] dénouement», un «temps subjectivisé ${ }^{22} »$. Ce temps est en fait celui du réel, car demander au principal protagoniste de rejouer des scènes du film presque trente ans plus tard fait reposer la narrativité sur la durée du temps réel. L'action déborde ainsi du temps fictif, sur laquelle le film dirige l'attention

20. Jan Estep, «An Interview with Pierre Huyghe», p. 32.

21. Jan Estep, «An Interview with Pierre Huyghe», p. 33.

22. Jean-Charles Masséra, «La leçon de Stains (Pour une esthétique de la reconstitution)», p. 48 . 
du début jusqu'à la fin pour entrer dans le cadre cinématographique, pour se réactualiser et s'inscrire à nouveau dans la vie de John Wojtowicz.

Le projet à l'origine de The Third Memory visait «à prolonger un récit, à ajouter un supplément d'histoire à un moment décrit, le film, en envisageant une série de récits parallèles d'après une articulation dans l'histoire initiale ${ }^{23}$ ». Le point de départ est un modèle défini, un film appartenant au genre du cinéma vécu. Le remake permet de sortir de ce territoire narratif pour prolonger la fiction dans le réel. La narration passe ainsi d'une structure à une absence de structure. En plus de remettre en question le modèle cinématographique en le confrontant à ses limites, le processus d'intermédialité met aussi en présence plusieurs narrativités qui, à leur tour, engagent d'autres prolongements qui viennent s'ajouter au film et agir en périphérie. Le récit de John Wojtowicz se construit en effet de façon transversale, en passant par différents formats et médias et en mettant en jeu plusieurs moments du temps. Avant d'entrer dans la salle de double projection vidéo, où les scènes refilmées voisinent quelques scènes du film original, on traverse d'abord une petite salle où sont réunis plusieurs documents: des articles de journaux relatant les faits saillants du hold-up; des images filmées sur les lieux réels au moment de la prise d'otages et qui avaient été diffusées en direct sur plusieurs chaînes de télévision; le commentaire de John Wojtowicz adressé au producteur d'Hollywood; puis son interview à la PBS enregistrée de la prison. Ces récits ont en commun de faire partie de l'histoire de John Wojtowicz.

$\mathrm{Si}$ les remakes décortiquent les mécanismes internes des systèmes narratifs dont ils sont la reprise, c'est pour les déplacer et les «libérer» en créant d'autres réseaux de relation. S'inscrivant en continuité avec cette idée, Two Minutes Out of Time (2000) renvoie explicitement à l'univers du jeu. En collaboration avec Philippe Parreno, il a acheté les droits d'une figure manga d'une agence japonaise se spécialisant dans la vente de caractères fictifs destinés à des compagnies de production qui réalisent des jeux vidéos, des mangas ou des films publicitaires. Ce geste avait pour but de libérer la figure du marché de la fiction et de ses industries. C'est ainsi que Pierre Huyghe et Phillippe Parreno ont, à partir d'un catalogue, acheté et acquis les droits d'une figure sans nom, une simple image bidimensionnelle, sans biographie et sans qualités et dont la possibilité de vie, voire de survie est limitée. À partir de là, ils ont proposé à plusieurs artistes de reprendre la figure et de raconter son histoire. Ainsi Two Minutes Out of Time est née pour se développer en un projet collectif, exponentiel, permettant tout d'abord de libérer la figure originale de la fiction pour donner ensuite une extension à son histoire. Cette figure imaginaire, à qui les deux artistes ont donné

23. Pierre Huyghe, texte inédit de la présentation de son projet de travail. Source: document d'archives du Centre Pompidou, Paris. 
le nom d'Annlee, s'est ainsi métamorphosée à l'intérieur de plusieurs œuvres (fig. 5). Sortie de la fiction, elle entre dans un autre monde, un monde de récits qui prolifèrent dans une zone instable entre fiction et réalité. Si dans chacun des remakes la question de la narrativité est implicitement liée à une critique de l'industrie cinématographique, ici ce sont les impératifs commerciaux du jeu qui sont perçus comme exerçant une emprise sur la fiction, sur l'imaginaire.

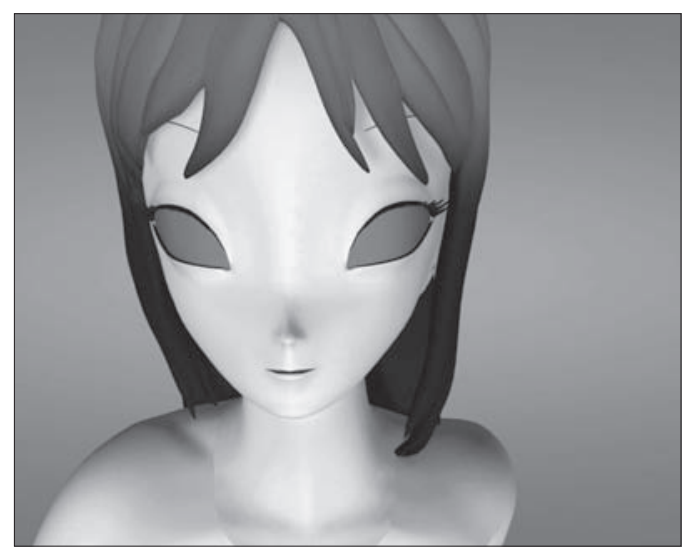

Fig. 5. Pierre Huyghe, Two minutes Out of Time, 2000, Beta digital, 4 minutes. Avec l'aimable permission de la Galerie Marian Goodman, New York/Paris.

Ces prolongements n'indiquent pas un renoncement au principe du récit. Au contraire, ils génèrent des reconstitutions narratives qui viennent ajouter d'autres interprétations, qui le projettent virtuellement. La narrativité n'est donc pas affaiblie ou inopérante, elle prend plutôt de l'expansion et devient proliférante. Les jeux sur la narrativité que produit le remake tendent donc à faire émerger d'autres récits, à ouvrir de multiples perspectives d'interprétation et, sur le plan idéologique, à libérer la représentation du créneau hollywoodien. Cette prolifération est possible si la narrativité travaille le mouvement d'un point à un autre, non le point lui-même, pour se développer «inchoativement», extensivement dans les interstices, dans un mouvement de balancier qui circule dans un sens comme dans une autre, et d'une temporalité à une autre. L'idée de prendre un film pour en proposer une «nouvelle» (une «autre») version permet d'étendre sa temporalité et d'en prolonger le récit parce que ce geste de reprise - et de réappropriation également - lui confère une mobilité. 